$63 \%$ of the UK\&I centres a pain tool is used but there was wide variation of what tools were used across centres. In contrast, $100 \%$ of the NL centres use a pain tool, in $75 \%$ the COMFORT Behavioural Scale and Visual Analogue scale or Numeric Rating Scale was used. Assessment tools for the cognitively impaired children were used in $57 \%$ of the UK\&I centres, however not in NL centres.

Sedation tools were used in $66 \%$ and $62 \%$, respectively (UK\&I and NL) centres. The most common tool is the COMFORT scale.

Regarding drug withdrawal, $71 \%$ of UK\&I centres did not have specific guidelines. However, $50 \%$ of the NL centres have specific guidelines and $75 \%$ of centres used a withdrawal tool.

Conclusions Although there are similarities between UK\&I and NL in pain, sedation and withdrawal assessment and management, there is more focus on sedation and withdrawal in the NL compared to that of a stronger pain focus in the UK\&I.

\section{VACCINATION OF TURKISH INFANTS, CHILDREN \& ADOLESCENTS; PRACTICE BASED APPROACH}

doi:10.1136/archdischild-2012-302724.0092

D Gözen. Pediatric Nursing, Istanbul University Florence Nightingale Faculty of Nursing, Istanbul, Turkey

Infection diseases are cause of serious morbidity and mortality in children and adults. Immunization is the most cost-effective interventions for child survival and well being recommended by World health Organization (WHO). The "Expanded Programme on Immunization"(EPI) was established by WHO in 1974. Global Immunization Vision Strategy (GIVS) was developped by UNICEF and WHO in 2005 which aims to decrease morbitidy and mortality from preventable diseases by immunization programmes.

Immunization rates increased over $90 \%$ in Turkey by national vaccination Schedule in recent years. Vaccines in National vaccination Schedule are practiced free in the Family Health Centers by Ministry of Health for all children in Turkey.

Immunization for children varies and regulated depending on child's physical and functional condition. The true way and niddle angle of the vaccine injection is very important. In the literature it is recommended that update the knowledge of the nurses about vaccine administration is effet to decrease side effects of vaccines.

The Mininistry of Health's one of the objective of "Healthy Society 2010 " is increase immunization rates over $90 \%$ for adolescents. Adolescent Health Centers evaluate immunization histories of all adolescents and recommend vaccines for missed vaccinated adolescents. Human Papilloma Virus (HPV) vaccine is recommended for adolescents to protect them from servical cancers. Because of HPV vaccine doesnt placed in the National Childhood Vaccination Schedule. Hepatit B, combined diphtheria, tetanus, aselular pertusis, measles, mumps and rubella, varicella, meningococcal vaccine, and HPV vaccine application is recommended for 11-18 years of age in the routine vaccination Schedule all adolescents.

\section{HOW CAN WE PREVENT VENTILATOR-ASSOCIATED PNEUMONIA IN PICU?}

doi:10.1136/archdischild-2012-302724.0093

D Sönmez Düzkaya. Istanbul University, Istanbul Faculty of Medicine Directorate of Nursing Services, Supervisor Nurse, Istanbul, Turkey

Ventilator-associated pneumonia (VAP) is a nosocomial infection that develops in patients on mechanical ventilation for $48 \mathrm{~h}$ or more. VAP has been classified into either early-onset pneumonia $(\mathrm{EOP})$, if pneumonia develops within 4 days of the patient's admission to an ICU or intubation for mechanical ventilation, and late-onset pneumonia (LOP), if pneumonia develops after 4 days of the patient's admission to an ICU or intubation for mechanical ventilation.

VAP is the second most common hospital-acquired infection among pediatric and neonatal intensive care unit (PICU) (NICU) patients. VAP is associated with increased morbidity and mortality rates, prolongs hospital length of stay (LOS) and increases medical costs. VAP is the most frequentandcostly infectious complication in ICU patients, which has been estimated to cost at least $\$ 40,000$ per patient as estimated in 3 matched cohort studies.

Studies provide preliminary information on risk factors that may be associated with the development of pediatric VAP. These include length of mechanical ventilation, use of opiates, sustained neuromuscular blockade, presence of enteral nutrition, prior antibiotic therapy, endotracheal suctioning, reintubation, gastroesophageal reflux, subglottal/tracheal stenosis, age greater than 10 years, and trauma.

Strategies for preventing or controlling ventilatorassociated pneumonia;

- Hand decontamination and use of gloves

- Head-of-bed elevation

- Oral/nasal hygiene

- Daily sedation vacation and daily assessment of readiness to extubate

- In-Line Suctioning

- The Bundle Approach

- Peptic ulcer prophylaxis

- Subglottic secretion drainage

- Orotracheal v. nasotracheal intubation

- Enteral feeding and control of regurgitation

\section{4 IMMUNOMODULATION IN SEPSIS: MORE HARM THAN GOOD?}

doi:10.1136/archdischild-2012-302724.0094

\section{S Opal. Infectious Disease Division, Memorial Hospital of RI, Pawtucket, RI, USA}

The systemic inflammatory response that typifies septic shock can induce widespread tissue injury, endothelial dysfunction and lethality. Interventions that disrupt the systemic inflammatory response have been the central treatment strategy for septic shock for decades. In experimental models, inhibitors of generalized inflammation are of clear survival benefit when the inducing stimulus is LPS or other bacterial toxins. However, the response to inhibitors of inflammation in actual infection models such as pneumonia, peritonitis or soft tissue infections generate variable results with improvement in some animal models and harm in others. Intrinsic counter regulatory mechanisms that accompany systemic inflammation can induce a prolonged phase of relative immune suppression. Attempts to restore immune function by immunoadjuvants have become a potential therapy for sepsis. Genomic evidence indicates that activation of innate immunity and depression of adaptive immunity occurs simultaneously in the very early phases of septic shock. The magnitude of the change from resting state is the most predictive outcome rather than specific patterns of immune dysfunction. Non-resolving information poses a real challenge from a therapeutic prospective in patients with septic shock. A new generation of anti-inflammatory agents and anticoagulants are now in clinical development along with a variety of immunostimulants that promote adaptive immune function and inhibitors of negative regulators of inflammation such as programed cell death (PD-1) or B and $\mathrm{T}$ lymphocyte attenuator. These interventions are in clinical trials, and the results will determine the direction in which experimental therapeutics take in the near future in the management of septic shock. 